\title{
Enhanced Direct Conversion of Fibroblasts into Hepatocyte-Like Cells by Kdm2b
}

Kobra Zakikhan ${ }^{1}$, Behshad Pournasr ${ }^{2}$, Marjan Nassiri-Asl ${ }^{3}$, Hossein Baharvand $^{2,4^{*}}$

1. Department of Molecular Medicine, School of Medicine, Qazvin University of Medical Sciences, Qazvin, Iran

2. Department of Stem Cells and Developmental Biology, Cell Science Research Center, Royan Institute for Stem Cell Biology and Technology, ACECR, Tehran, Iran

3. Cellular and Molecular Research Center, Department of Pharmacology, School of Medicine, Qazvin University of Medical Sciences, Qazvin, Iran

4. Department of Developmental Biology, University of Science and Culture, ACECR, Tehran, Iran

\section{*Correspondence:}

Hossein Baharvand, $\mathrm{PhD}$

Tel: +98 2122306485

Fax: +982123562507

Email: Baharvand@RoyanInstitute.org

Running title: Enhanced conversion of fibroblasts into iHep cells by $K d m 2 b$ 


\begin{abstract}
Cell fate conversion of terminally differentiated cells by defined transcription factors between different lineages is a new approach to produce new cells that have the capability to repair or replace diseased and damaged tissues. Previous studies have demonstrated that this inefficient process can be facilitated by the inclusion of additional factors. Here we report that $K d m 2 b$, a histone demethylase, has the capability to promote conversion of fibroblasts to functional induced hepatocyte-like (iHep) cells in combination with previously reported hepatic lineage transcription factors, $H n f 4 \alpha$ and Foxa3. This approach led to increased numbers of epithelial-like colonies, hepatic markers and functionality which included periodic acid-Schiff (PAS) positive colonies, CYP450 activity, low-density lipoprotein and indocyanin green (ICG) uptake, as well as Albumin secretion. Additionally, the transplanted iHep cells were engraftable, expressed Albumin, and contributed to the recovery of a carbon tetrachloride-injured mouse model. These results have not only identified an important epigenetic factor for iHep generation, but also brought new insight into the molecular nature of hepatogenesis and future biomedical applications for liver diseases.
\end{abstract}

Keywords: Direct conversion; Induced hepatocyte-like cells; $K d m 2 b$; Transdifferentiation 


\section{Introduction}

Direct lineage conversion or trans-differentiation of cells is a new technology that has the capability to generate functional cells for studies of basic science and clinical applications. This method is also known as transdifferentiation. Transdifferentiation is a type of lineage reprograming that directly switches one mature somatic cell type into another functional somatic cell type or progenitor, primarily by ectopic expression of lineage-instructive defined transcription factors (TFs) without passing through the pluripotent state $[1,2]$. Numerous reports discuss the conversion of mice and human fibroblasts (for review see [3, 4]). In these studies, direct conversation of fibroblasts to induced hepatocyte-like cells (iHep) has been performed with the following combinations: Hnf4a plus Foxal, Foxa2 or Foxa3 [5]; Hnfla plus Gata4 and Foxa3, along with inactivation of $\mathrm{p} 19^{\mathrm{Arf}}[6] ; H N F 1 \alpha, H N F 4 \alpha$, and $H N F 6$ along with maturation factors ATF5, PROX1, C/EBP $\beta$ and p53 shRNA [7]; FOXA3, HNF1 $\alpha$, and $H N F 4 \alpha$ [8]; or FOXA2, HNF4 $\alpha, C / E B P \beta$ and $C$-MYC [9]. Moreover, Hnflb and Foxa3 are sufficient to reprogram mouse fibroblasts into induced hepatic stem cells [10]. Another strategy that resulted in hepatic reprogramming used Yamanaka factors to generate epigenetic instability in conjunction with small molecules [11] and/or a cocktail of Hnf4a, Cebpa and Nrli2 [12] under hepatic conditions. Nevertheless, the approaches that increase the direct conversion into a hepatic fate should be taken into consideration. Numerous factors has been reported to affect reprogramming efficiency through epigenetic modification and cell-cycle-dependent or independent mechanisms (for review see [13-15]). For example, inhibition of the p53-p21 pathway and the Arf locus can increase reprogramming efficiency and accelerate hepatic reprogramming by affecting cell proliferation $[6,7]$. 
A recent study has reported that $K d m 2 b$ (also known as $J h d m 1 b$ and Fbxllo), an H3K36me2 (histone H3 Lys 36 dimethyl)-specific demethylase, can facilitate induced pluripotent stem (iPS) cell generation independent of its effect on cell proliferation [16, 17]. However, whether this factor is involved in hepatic reprogramming remains unknown.

We sought to determine whether $K d m 2 b$ increased the transdifferentiation efficiency of mouse fetal limb fibroblasts to convert into a hepatic lineage. This study examined $K d m 2 b$ overexpression in hepatic induction of the fibroblasts when used in conjunction with $H n f 4 \alpha$ and Foxa 3 [5]. The results indicated that $K d m 2 b$ increased hepatic marker expressions, along with improved functionality of the iHep cells and enabled them to engraft after transplantation into the mice with carbon tetrachloride-induced acute liver damage.

\section{Materials and methods}

\subsection{Virus production and direct hepatic conversion}

ViraPower ${ }^{\mathrm{TM}}$ pLenti3.6/TO/V5-DEST backbone was purchased from Invitrogen. We separately subcloned synthetized $H n f 4 \alpha, F o x a 3$ and $K d m 2 b$ into this vector. We used a maxiprep kit for plasmid preparation of all the genes which were subsequently stored at $-20^{\circ} \mathrm{C}$. Lentivirus production and titration were performed according to previously reported protocol [12] with some modifications. Briefly, the constructed plasmids were introduced into 293FT cells with 2 packaging plasmids, psPAX2 (Addgene, 12260) and an envelope plasmid (pMD2.G; Addgene, 12259) using lipofectamine 3000 (Invitrogen). After 48 and $72 \mathrm{~h}$ incubation periods, the medium that contained the lentiviruses was collected and passed through a $0.45 \mu \mathrm{m}$ filter. The viruses were kept at $-70^{\circ} \mathrm{C}$. The $\mathrm{HT} 1080$ cell line was used for titration according to the manufacturer's protocol. 
Transduction was performed by infecting approximately $1 \times 10^{6}$ suspended mouse fibroblasts derived from 12.5 day-old mouse fetal limbs with the lentiviruses in the presence of $6 \mu \mathrm{g} / \mathrm{ml}$ polybrene (Sigma-Aldrich), without antibiotic and serum. We renewed the medium one day after transduction. At two days after transduction, the transduced cells were incubated in hepatic medium at $37^{\circ} \mathrm{C}$ (Fig. 1A). The medium was changed every other day.

\subsection{Cell culture, hepatic media, immunostaining, quantitative RT-PCR (qRT-PCR), and functional analyses}

It has been described in details in supplementary Materials and methods.

\section{Results}

\subsection{Reprogramming of fibroblasts by hepatic specific transcription factors (TFs)}

Figure 1A shows the experimental scheme for direct conversion of mouse fibroblasts into iHep cells. We prepared fibroblasts from mouse fetal limbs in order to avoid contamination by cells from organs of the digestive system. Hnf4a and Foxa3 TFs were chosen because of their importance in liver development and function. These TFs have been reported to be an effective combination of factors for hepatic reprogramming [5]. In order to increase hepatic reprogramming efficiency and function, we assessed the effects of $K d m 2 b$ which facilitated mesenchymal-to-epithelial transition (MET) [16, 17]. Mixtures of the lentiviral vectors that encoded Hnf4 $\alpha$ and Foxa3 (HF group) or Hnf4 $\alpha$, Foxa3 and $K d m 2 b$ (HFK group) were used to infect mouse fibroblasts for one day. On day 2, the cells were cultured in fibroblast medium. From day 2 we changed the medium to hepatic medium 1 (HM1) that consisted of dexamethasone (Dex), nicotinamide (Nic), hepatocyte growth factor (HGF), epidermal growth 
factor (EGF), oncostatin M (OSM), and A-83-01 (A83). A83, a transforming growth factor-b type I (TGF- $\beta$ ) receptor inhibitor, has been shown to prevent mesenchymal signals during treatment of cells with hepatic medium [11]. We observed that some cells gradually transformed into epithelial-like colonies from days 8-10 (Fig. 1B). We also used a modified hepatic medium (HM2) that consisted of bone morphogenetic protein (BMP4) and fibroblast growth factor 4 (FGF4) added to HM1. BMP4 was added for the first three days because of the undesirable effects on MET [18]. Under these circumstances, the epithelial-like colonies appeared earlier, from days 5-8 (Fig. 1C). We observed a reduction in the number and size of the colonies grown in HM1 after day 11. However, colonies grown in HM2 showed gradual transformation into a polygonal shape with distinct round nuclei and large cytoplasms (Fig. 1D). We designated these cells, iHep cells.

Evaluation of epithelial-like colony numbers and the total surface area of the colonies in both media for HF and HFK showed that the parameters increased significantly in HM2 (at least $P<0.05$, Fig. 1E and F). Application of HFK resulted in significant enhancement in the numbers and total surface area of the colonies (at least $P<0.05$, Fig. 1E and F). Therefore, we used HM2 to reprogram fibroblasts into a hepatic lineage.

\section{2. $K d m 2 b$ combined with hepatic-lineage factors enhanced the conversion of fibroblasts to}

\section{a hepatic fate}

Next, we sought to determine if $K d m 2 b$ combined with $H n f 4 \alpha$ and Foxa 3 TFs could enhance iHep cells at day 11 after infection. As seen in Figure 2A, gene expression analysis with realtime PCR revealed that overexpression of $K d m 2 b$ resulted in significant upregulation of Transthyretin (Ttr), Tyrosine aminotransferase (Tat), Hepatocyte nuclear factor 4 (Hnf $4 \alpha$ ), 
Asialoglycoprotein receptor (Asgpr), Glucose-6-phosphatase (G6p), GATA binding protein 4

(Gata4), and E-cadherin (Cdhl) (Fig. 2A, at least $P<0.05$ ). However, the expressions of Albumin (Alb) and the Cytochrome P450-family 2-subfamily b-polypeptide 9 (Cyp2b9) did not significantly differ (Fig. 2A).

Further immunofluorescence analysis of the HFK group showed that hepatocyte specific proteins Alb, Cyp3A4 and Hnf4 $\alpha$ expressed in this group (Fig. 2Bi). iHep cells also expressed E-cadherin as an epithelial marker. A number of cells co-expressed Asgpr as a mature specific hepatocyte marker (Fig. 2Bii). As seen in Figure 2Biii, we observed detectable levels of the hepatic progenitors Leucine-rich repeat-containing G-protein coupled receptor 5 (Lgr5), Delta-like 1 homolog (Dlk1), and Epithelial cell adhesion molecule (EpCAM).

\subsection{Hepatic specific functions in iHep cells}

PAS staining revealed glycogen storage in iHep cells (Fig. 3A and Supplementary Fig. 1). The HFK group had a higher percentage of PAS-positive colonies compared to the HF group (Fig. 3B, $P<0.05)$. With regards to hepatocyte properties, the HFK-iHep cells were competent for CYP3A4-mediated 7-pentoxyresorufin O-dealkylase (PROD) activity as demonstrated after phenobarbital induction. Fluorescence intensity of the cells increased after phenobarbital induction in HFK compared to HF (Fig. 3D, $P<0.05$ ). We evaluated cellular uptake of indocyanin green (ICG), a nontoxic organic anion clinically used to assess liver function. This anion is eliminated exclusively by hepatocytes. As shown in Figure 3E and Supplementary Figure 1, iHep cells incorporated ICG and had the capability to uptake fluorescent LDL. In addition, we analyzed the cells for their ability to secret Alb in culture. The ELISA results 
showed higher significant levels of Alb in the HFK group compared to the HF group (Fig. 3F, $P<0.05)$. These results indicated the important functions of functional iHep cells.

In order to investigate whether the reprogrammed cells could be engrafted, we transplanted $1 \times$ $10^{6}$ DiI-labeled cells into the spleens of cyclosporine-treated mice that had acute $\mathrm{CCl}_{4}$ liver damage. Animal survival was evaluated up to two weeks after $\mathrm{CCl}_{4}$ injection. In the HFK-iHep cell transplanted group, $100 \%$ of the animals survived compared to $80 \%$ of the animals in the fibroblast transplanted group. At day 14, we observed DiI-labeled HFK-iHep cells in the recipient livers by fluorescent microscope (Fig. 3G). Most of the engrafted cells were detected around the pericentral and periportal veins of the hepatic lobules. In an attempt to determine their fate, we immunostained the mice liver sections for Alb and DiI positive cells to assess Alb expression. Double DiI/Alb-positive cells were considered an indicator of functional activity of the transplanted iHep cells.

Taken together, these findings showed that HFK-iHep cells grown in HM2 medium exhibited a number of specific morphological and functional features of hepatocytes.

\section{Discussion}

In this study, we demonstrated that $K d m 2 b$ as an epigenetic modifier with two hepatic lineage factors, combined with $H n f 4 \alpha$ and Foxa3 [5] in mouse fibroblasts grown in hepatic medium significantly enhanced hepatic reprogramming. $K d m 2 b$ had a higher rate of epithelial-like colony formation compared with HF factors grown in HM2 medium compared to HM1 medium [5]. HM2 medium contained BMP4 and FGF4, two growth factors necessary during liver development (for review see $[19,20]$ ) and hepatocyte differentiation in vitro [21-23]. 
The HFK-reprogrammed cells expressed higher levels of the hepatic markers Ttr, Tat, Hnf4 $\alpha$, Asgpr, G6p, Gata4 and Cdhl and increased functionality as shown by the greater numbers of PAS positive colonies, increased CYP450 activity, ICG and LDL uptake, and Alb secretion. We transplanted the HFK-iHep cells into a $\mathrm{CCl}_{4}$-injured mouse model and observed that the reprogramming cells engrafted, expressed Alb, and exhibited functionality in vivo by contributing to the recovery of the mouse model compared to transplanted fibroblasts. We named these reprogrammed iHep cells, as neither a fully immature or fully mature state could be determined. Advantages of direct conversion have included the lack of tumorigenic risk, rapid conversion rate, and repair of injured tissues by in vivo reprogramming (for review see $[3,4$, 24]).

It has been shown that $K d m 2 b$ can accelerate conversion of fibroblasts to iPS cells in conjugation with Yamanaka factors $[16,17] . K d m 2 b$ has been shown to contribute to gene activation by binding to and demethylating the gene promoters, increasing epithelialization and facilitating the activation of early responsive genes during reprogramming [16]. We observed that $K d m 2 b$ mediated upregulation of epithelial markers Cdh1 and EpCAM. The expression of $K d m 2 b$ as like as transient presence of BMP4, TGF- $\beta$ inhibitors and microRNA-200s facilitating MET, and promotes iPS cell generation [25-28]. According to research, activation of an epithelial transcription program early in reprogramming was crucial for the generation of pluripotency and rapid conversion of fibroblasts to iHep cells. Researchers reported that $K d m 2 b$ interacted with the core components of polycomb repressive complex 1 and recruited this complex to the $\mathrm{CpG}$ islands of early lineage-specific genes [29]. A novel approach for RNA-sequencing showed that $K d m 2 b$ expressed at much higher levels in mouse perinatal livers compared to adult livers [30]. This suggested that ontogenic changes in mRNA expression of epigenetic modifiers such as 
$K d m 2 b$ might have crucial roles in determining the addition and/or removal of corresponding epigenetic signatures during liver development [30].

The findings presented here, for the first time, have shown that the application of an epigenetic modifier, $K d m 2 b$, combined with two hepatic factors increased efficient reprogramming of mouse fibroblasts into functional iHep cells. Future metabolic and in vivo characterizations are necessary to identify the maturity and function of these iHep cells. It remains to be determined if this combination can produce iHep cells from human somatic cells. However, similar to other cell-fate reprogramming studies, our results may open another window to develop biomedical strategies for liver diseases.

\section{Acknowledgments}

This study was funded by grants provided from Royan Institute, the Iranian Council of Stem Cell Research and Technology, the Iran National Science Foundation (INSF), and Iran Science Elites

Federation to H.B. We express our appreciation to all members of the Hepatocyte Program at Royan Institute for their helpful deliberation and consultation during this work. We express our appreciation to Dr. Mohammad Hassan Asghari-Vostikolaee for his assistance in performing animal studies. 


\section{References}

[1] B. Pournasr, K. Khaloughi, G.H. Salekdeh, M. Totonchi, E. Shahbazi, H. Baharvand, Concise review: alchemy of biology: generating desired cell types from abundant and accessible cells, Stem cells, 29 (2011) 1933-1941.

[2] I. Sancho-Martinez, S.H. Baek, J.C. Izpisua Belmonte, Lineage conversion methodologies meet the reprogramming toolbox, Nature cell biology, 14 (2012) 892-899.

[3] F. Mirakhori, B. Zeynali, G.H. Salekdeh, H. Baharvand, Induced neural lineage cells as repair kits: so close, yet so far away, J Cell Physiol, 229 (2014) 728-742.

[4] T. Vierbuchen, M. Wernig, Direct lineage conversions: unnatural but useful?, Nat Biotechnol, 29 (2011) 892-907.

[5] S. Sekiya, A. Suzuki, Direct conversion of mouse fibroblasts to hepatocyte-like cells by defined factors, Nature, 475 (2011) 390-393.

[6] P. Huang, Z. He, S. Ji, H. Sun, D. Xiang, C. Liu, Y. Hu, X. Wang, L. Hui, Induction of functional hepatocyte-like cells from mouse fibroblasts by defined factors, Nature, 475 (2011) 386-389.

[7] Y. Du, J. Wang, J. Jia, N. Song, C. Xiang, J. Xu, Z. Hou, X. Su, B. Liu, T. Jiang, D. Zhao, Y. Sun, J. Shu, Q. Guo, M. Yin, D. Sun, S. Lu, Y. Shi, H. Deng, Human hepatocytes with drug metabolic function induced from fibroblasts by lineage reprogramming, Cell stem cell, 14 (2014) 394-403.

[8] P. Huang, L. Zhang, Y. Gao, Z. He, D. Yao, Z. Wu, J. Cen, X. Chen, C. Liu, Y. Hu, D. Lai, Z. Hu, L. Chen, Y. Zhang, X. Cheng, X. Ma, G. Pan, X. Wang, L. Hui, Direct reprogramming of human fibroblasts to functional and expandable hepatocytes, Cell stem cell, 14 (2014) 370-384.

[9] T. Kogiso, H. Nagahara, M. Otsuka, K. Shiratori, S.F. Dowdy, Transdifferentiation of human fibroblasts into hepatocyte-like cells by defined transcriptional factors, Hepatology international, 7 (2013) 937-944.

[10] B. Yu, Z.Y. He, P. You, Q.W. Han, D. Xiang, F. Chen, M.J. Wang, C.C. Liu, X.W. Lin, U. Borjigin, X.Y. Zi, J.X. Li, H.Y. Zhu, W.L. Li, C.S. Han, K.J. Wangensteen, Y. Shi, L.J. Hui, X. Wang, Y.P. Hu, Reprogramming fibroblasts into bipotential hepatic stem cells by defined factors, Cell stem cell, 13 (2013) 328-340.

[11] S. Zhu, M. Rezvani, J. Harbell, A.N. Mattis, A.R. Wolfe, L.Z. Benet, H. Willenbring, S. Ding, Mouse liver repopulation with hepatocytes generated from human fibroblasts, Nature, 508 (2014) 93-97.

[12] B. Pournasr, M.H. Asghari-Vostikolaee, H. Baharvand, Transcription factor-mediated reprograming of fibroblasts to hepatocyte-like cells, European journal of cell biology, 94 (2015) 603-610. 
[13] A.J. Gruber, M. Zavolan, Modulation of epigenetic regulators and cell fate decisions by miRNAs, Epigenomics, 5 (2013) 671-683.

[14] J.H. Hanna, K. Saha, R. Jaenisch, Pluripotency and cellular reprogramming: facts, hypotheses, unresolved issues, Cell, 143 (2010) 508-525.

[15] M. Stadtfeld, K. Hochedlinger, Induced pluripotency: history, mechanisms, and applications, Genes Dev, 24 (2010) 2239-2263.

[16] G. Liang, J. He, Y. Zhang, Kdm2b promotes induced pluripotent stem cell generation by facilitating gene activation early in reprogramming, Nature cell biology, 14 (2012) 457-466.

[17] S.E. Vidal, B. Amlani, T. Chen, A. Tsirigos, M. Stadtfeld, Combinatorial modulation of signaling pathways reveals cell-type-specific requirements for highly efficient and synchronous iPSC reprogramming, Stem cell reports, 3 (2014) 574-584.

[18] N. McCormack, E.L. Molloy, S. O'Dea, Bone morphogenetic proteins enhance an epithelialmesenchymal transition in normal airway epithelial cells during restitution of a disrupted epithelium, Respiratory research, 14 (2013) 36.

[19] M. Gordillo, T. Evans, V. Gouon-Evans, Orchestrating liver development, Development, 142 (2015) 2094-2108.

[20] K.S. Zaret, M. Grompe, Generation and regeneration of cells of the liver and pancreas, Science, 322 (2008) 1490-1494.

[21] H. Basma, A. Soto-Gutierrez, G.R. Yannam, L. Liu, R. Ito, T. Yamamoto, E. Ellis, S.D. Carson, S. Sato, Y. Chen, D. Muirhead, N. Navarro-Alvarez, R.J. Wong, J. Roy-Chowdhury, J.L. Platt, D.F. Mercer, J.D. Miller, S.C. Strom, N. Kobayashi, I.J. Fox, Differentiation and transplantation of human embryonic stem cell-derived hepatocytes, Gastroenterology, 136 (2009) 990-999.

[22] K. Si-Tayeb, F.K. Noto, M. Nagaoka, J. Li, M.A. Battle, C. Duris, P.E. North, S. Dalton, S.A. Duncan, Highly efficient generation of human hepatocyte-like cells from induced pluripotent stem cells, Hepatology, 51 (2010) 297-305.

[23] D.C. Hay, S. Pernagallo, J.J. Diaz-Mochon, C.N. Medine, S. Greenhough, Z. Hannoun, J. Schrader, J.R. Black, J. Fletcher, D. Dalgetty, A.I. Thompson, P.N. Newsome, S.J. Forbes, J.A. Ross, M. Bradley, J.P. Iredale, Unbiased screening of polymer libraries to define novel substrates for functional hepatocytes with inducible drug metabolism, Stem Cell Res, 6 (2011) 92-102.

[24] U. Ben-David, N. Benvenisty, The tumorigenicity of human embryonic and induced pluripotent stem cells, Nat Rev Cancer, 11 (2011) 268-277.

[25] R. Li, J. Liang, S. Ni, T. Zhou, X. Qing, H. Li, W. He, J. Chen, F. Li, Q. Zhuang, B. Qin, J. Xu, W. Li, J. Yang, Y. Gan, D. Qin, S. Feng, H. Song, D. Yang, B. Zhang, L. Zeng, L. Lai, M.A. Esteban, D. Pei, A mesenchymal-to-epithelial transition initiates and is required for the nuclear reprogramming of mouse fibroblasts, Cell stem cell, 7 (2010) 51-63. 
[26] P. Samavarchi-Tehrani, A. Golipour, L. David, H.K. Sung, T.A. Beyer, A. Datti, K. Woltjen, A. Nagy, J.L. Wrana, Functional genomics reveals a BMP-driven mesenchymal-toepithelial transition in the initiation of somatic cell reprogramming, Cell stem cell, 7 (2010) 6477.

[27] J.K. Ichida, J. Blanchard, K. Lam, E.Y. Son, J.E. Chung, D. Egli, K.M. Loh, A.C. Carter, F.P. Di Giorgio, K. Koszka, D. Huangfu, H. Akutsu, D.R. Liu, L.L. Rubin, K. Eggan, A smallmolecule inhibitor of tgf-Beta signaling replaces sox 2 in reprogramming by inducing nanog, Cell stem cell, 5 (2009) 491-503.

[28] N. Maherali, K. Hochedlinger, Tgfbeta signal inhibition cooperates in the induction of iPSCs and replaces Sox2 and cMyc, Curr Biol, 19 (2009) 1718-1723.

[29] J. He, L. Shen, M. Wan, O. Taranova, H. Wu, Y. Zhang, Kdm2b maintains murine embryonic stem cell status by recruiting PRC1 complex to $\mathrm{CpG}$ islands of developmental genes, Nature cell biology, 15 (2013) 373-384.

[30] H. Lu, J.Y. Cui, S. Gunewardena, B. Yoo, X.B. Zhong, C.D. Klaassen, Hepatic ontogeny and tissue distribution of mRNAs of epigenetic modifiers in mice using RNA-sequencing, Epigenetics, 7 (2012) 914-929.

[31] Y. Tahamtani, M. Azarnia, A. Farrokhi, A. Sharifi-Zarchi, N. Aghdami, H. Baharvand, Treatment of human embryonic stem cells with different combinations of priming and inducing factors toward definitive endoderm, Stem cells and development, 22 (2013) 1419-1432.

[32] M. Vosough, E. Omidinia, M. Kadivar, M.A. Shokrgozar, B. Pournasr, N. Aghdami, H. Baharvand, Generation of Functional Hepatocyte-Like Cells from Human Pluripotent Stem Cells in a Scalable Suspension Culture, Stem cells and development, 22 (2013) 2693-2705. 


\section{Figure legends}

\section{Figure 1. Induction of hepatic fate by reprograming transcription factors (TFs) in hepatic}

media.

(A) Experimental design for the generation of induced hepatocyte-like cells (iHep). Limb fibroblasts were infected with lentiviral vectors that encoded $H n f 4 \alpha$ and Foxa3 (HF group) or $H n f 4 \alpha$, Foxa3 and $K d m 2 b$ (HFK group). These fibroblasts were cultured in either hepatic medium 1 (HM1) or hepatic medium 2 (HM2). HM1 consisted of dexamethasone (Dex, $\left.10^{-7} \mathrm{M}\right)$, nicotinamide (Nic, $10 \mathrm{mM}$ ), hepatocyte growth factor (HGF, $20 \mathrm{ng} / \mathrm{ml}$ ), epidermal growth factor (EGF, $20 \mathrm{ng} / \mathrm{ml}$ ), oncostatin M (OSM, $20 \mathrm{ng} / \mathrm{ml})$, and A-83-01 (A83, $0.5 \mu \mathrm{M})$. HM2 consisted of HM1 medium plus fibroblast growth factor 4 (FGF4, $20 \mathrm{ng} / \mathrm{ml})$ and bone morphogenetic protein (BMP4, $10 \mathrm{ng} / \mathrm{ml}$ ). BMP4 was used in the HM2 medium only during the first three days.

(B) and (C) Morphology of epithelial-like colonies at day 11 from the HF and HFK groups cultured in HM1.

(D) Morphology of an epithelial-like colony in the HFK group cultured in HM2. Under these conditions the cells gradually transformed into a polygonal shape with distinct round nuclei and large cytoplasms. However, the numbers and sizes of the colonies grown in HM1 medium decreased after day 11 .

(E) and (F) Colony numbers and total surface area of the colonies in both media and inductions at day 11. The numbers and total surface areas of the colonies increased significantly for the HF and HFK groups grown in HM2 medium compared to HM1. The HFK group showed significant enhancement of colony numbers and total surface area of the colonies.

Data are presented as mean \pm SD. Statistical analysis was determined by the student's $t$-test $(\mathrm{n}=8$ per group in HM1 and $\mathrm{n}=4$ per group in HM2); *: $P<0.05$. **: $P<0.01$. 
Figure 2. Gene expression analyses of induced hepatocyte-like (iHep) cells.

(A) qRT-PCR for induced hepatocyte-like (iHep) cells. The HFK-iHep cells showed higher expressions of hepatic markers Ttr, Tat, Hnf4a, Asgpr, G6p, Gata4 and Cdh1 compared to the HF-iHep cells. Data are presented as mean \pm SD. Statistical analysis was determined by the student's $t$-test (n=3 per group) at day 11. *: $P<0.05, * *: P<0.01, * *: P<0.001$. Comparative relative mRNA expression in iHep normalized to Gapdh and calibrated to mouse fibroblasts.

(B) Immunofluorescence staining of the HFK group at day 11 showed expression of (i) hepatocyte specific proteins Alb, Cyp3A4 and Hnf4a; (ii) E-cadherin (epithelial marker), Asgpr (mature hepatocyte marker); and (iii) hepatic progenitor markers Lgr5, Dlk1 and EpCAM. Nuclei were counterstained by DAPI.

Figure 3. Functional analyses of HFK-iHep cells.

(A) Glycogen storage by periodic acid-Schiff (PAS) staining (purple cytoplasm).

(B) Quantification of PAS positive colonies showed a significantly higher percent of colonies in the HFK-iHep group ( $\mathrm{n}=4$ per group).

(C) Fluorescence image of iHep cells indicative of 7-pentoxyresorufin O-dealkylase (PROD) activity.

(D) Fold change of fluorescence intensity in HFK-iHep cells after induction versus HF-iHep cells. PROD assessment of CYP3A4 activity after induction with phenobarbital. $\mathrm{n}=3$ per group.

(E) Indocyanin green (ICG) uptake (green color) and acetylated-LDL conjugated to DiI (DiI-AcLDL) uptake (red color) in iHep cells.

(F) Secretory albumin (Alb) protein levels as measured by ELISA. ( $\mathrm{n}=3$ per group) 
(G) Engrafted HFK-iHep cells in the mouse model of an injured liver. Transdifferentiated cells in the HFK-iHep group were labeled with DiI prior to injection in order to evaluate homing of the transplanted cells. We detected DiI-labeled cells pericentral and periportal vein of the $\mathrm{CCL}_{4}$ injected acute liver model two weeks after transplantation. Double DiI/Alb-positive cells were detected as a marker of functional activity of the transplanted HFK-iHep cells. The nuclei were counterstained with DAPI.

Data are presented as mean \pm SD. $*: P<0.05, * *: P<0.01$. All the experiments were performed at day 11. 
Figure 1

A

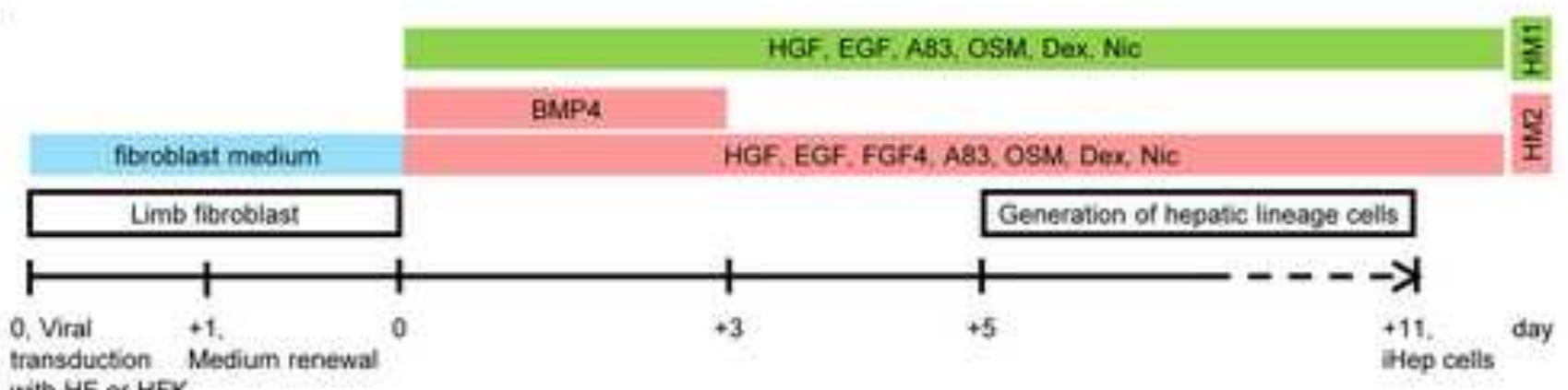

with HF or HFK

B

C
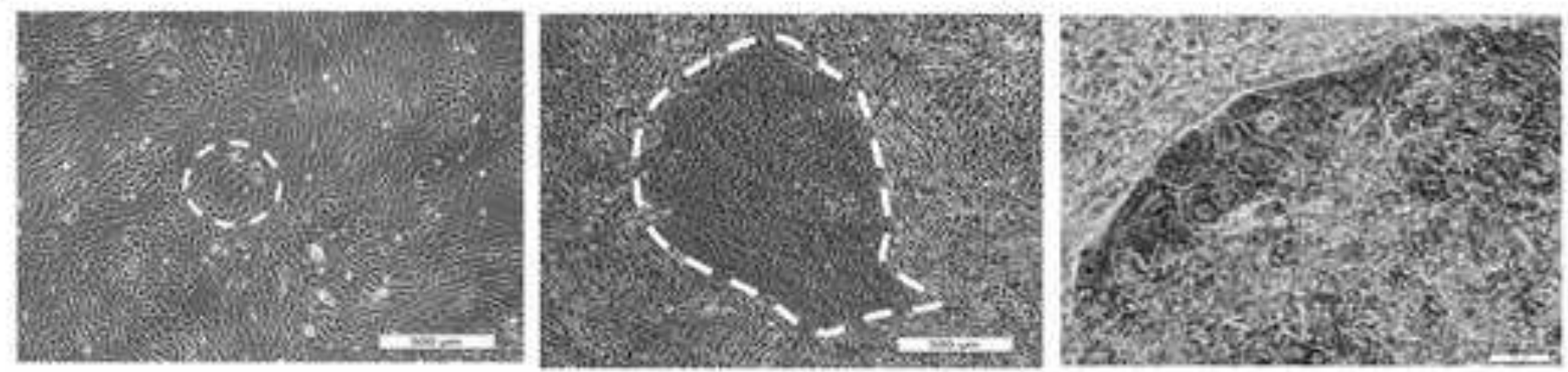

E

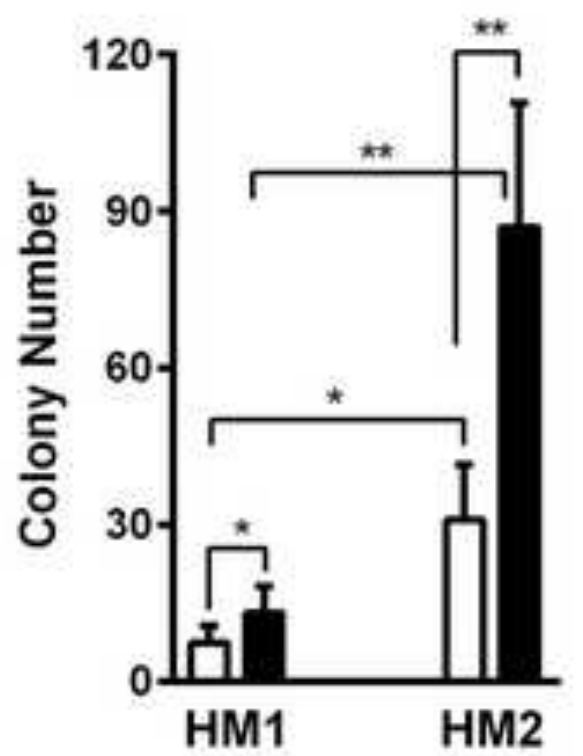

F

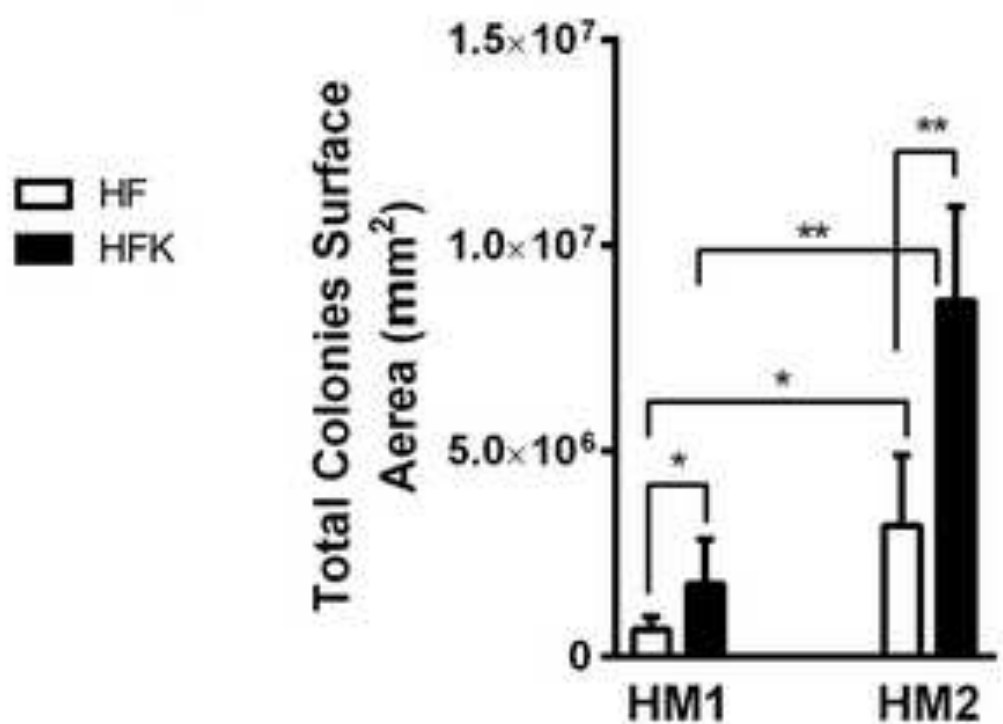




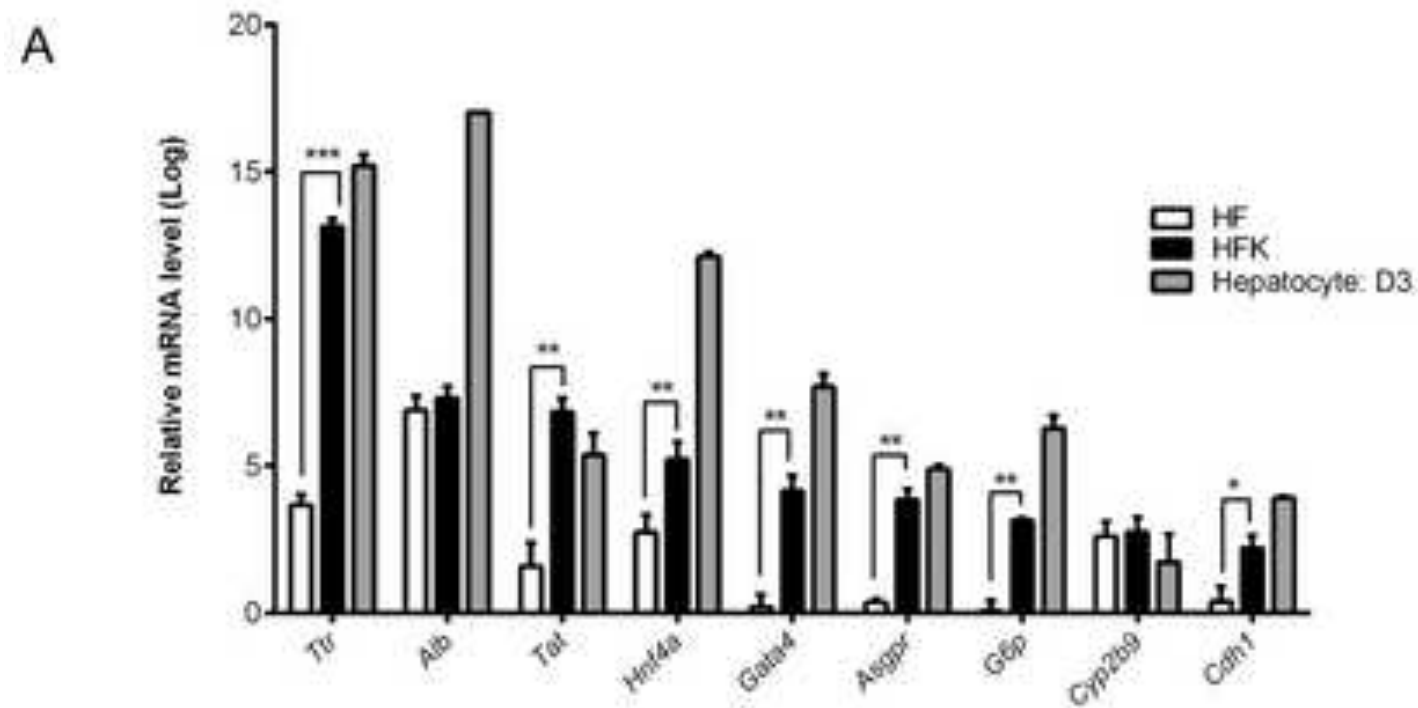

B (i)
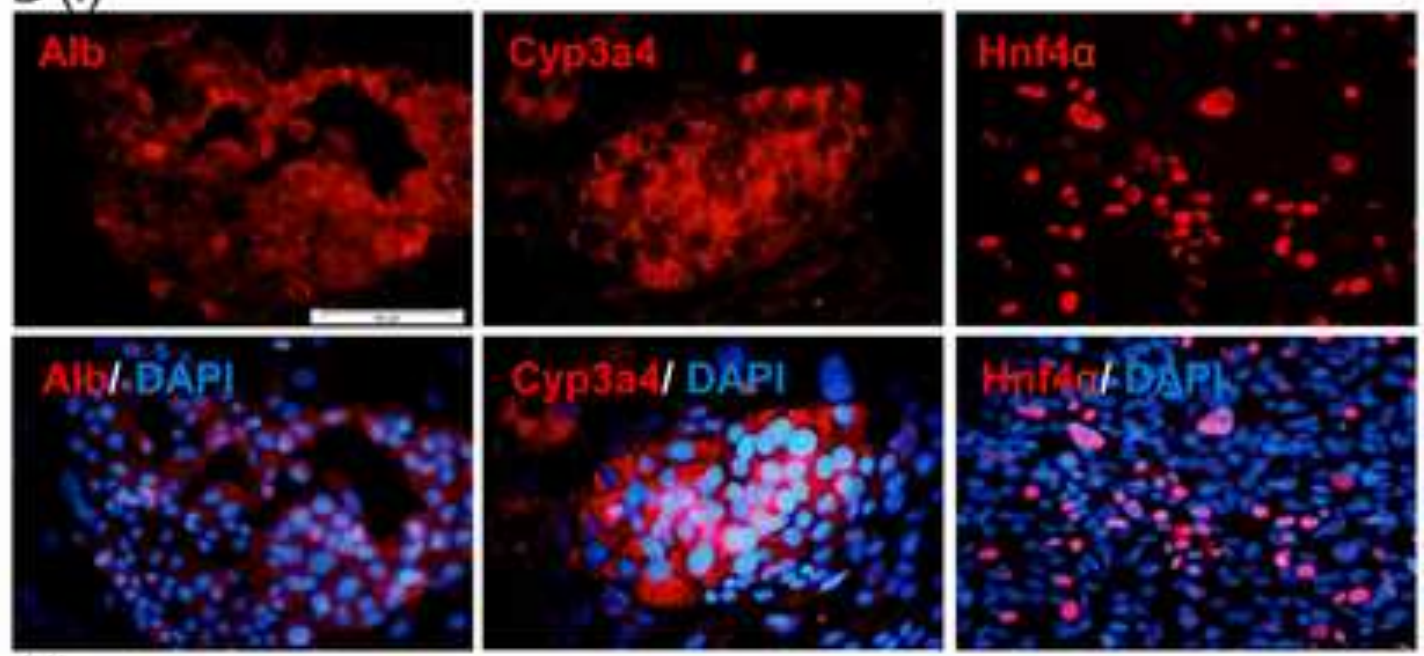

(ii)
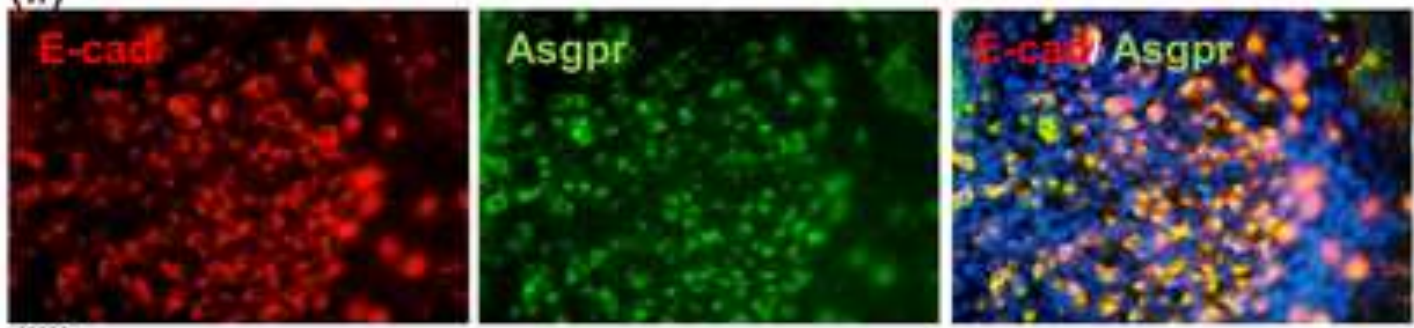

(iii)
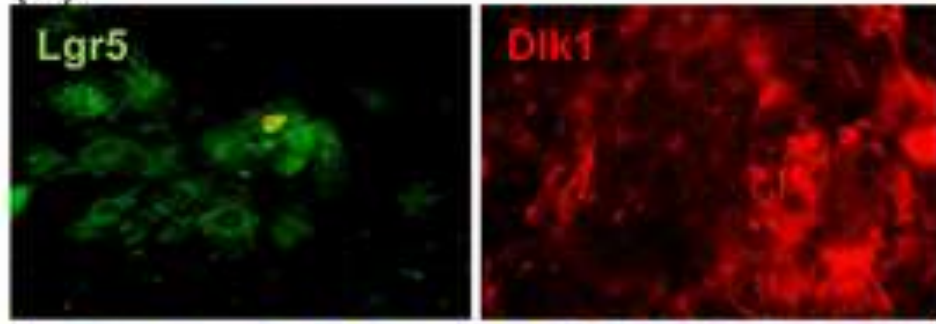

EpCAM
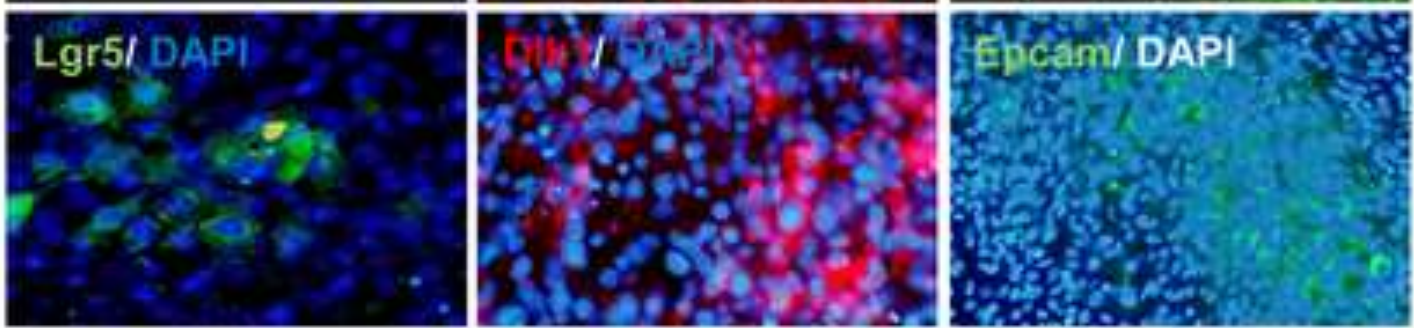
A
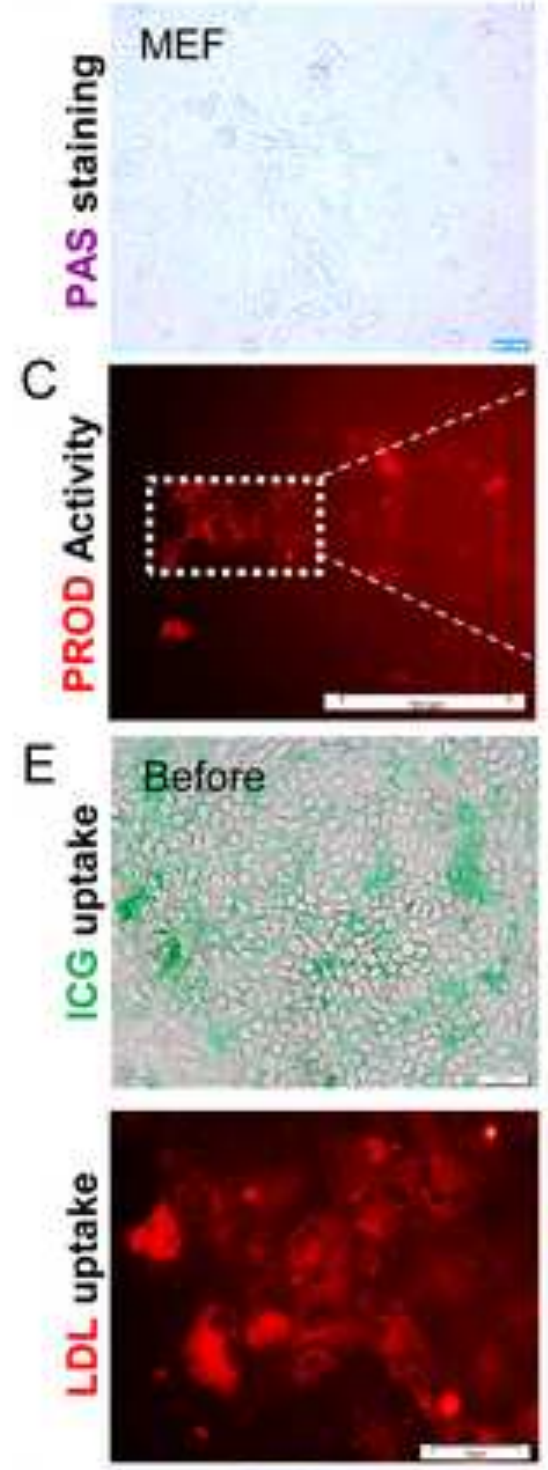

G

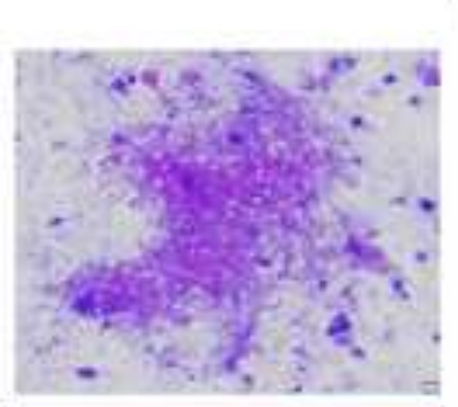

B
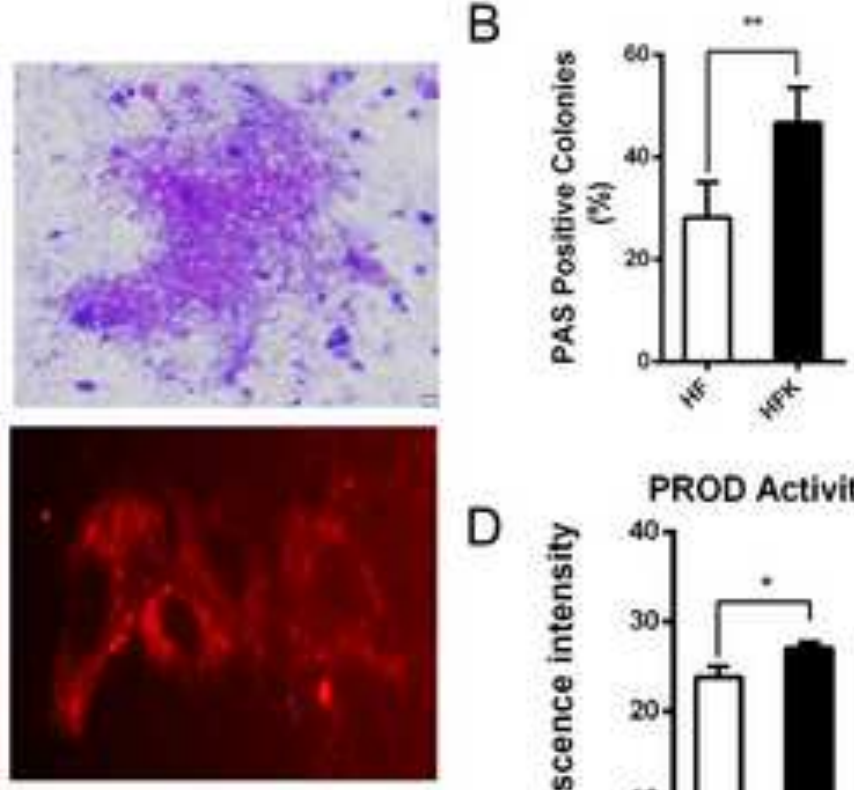

After

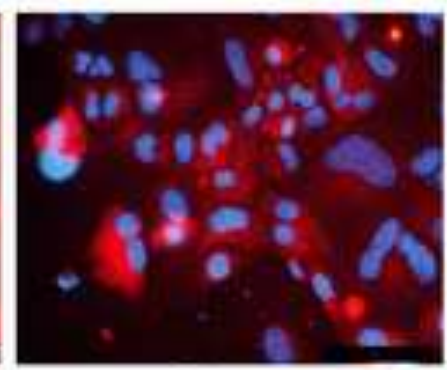

F

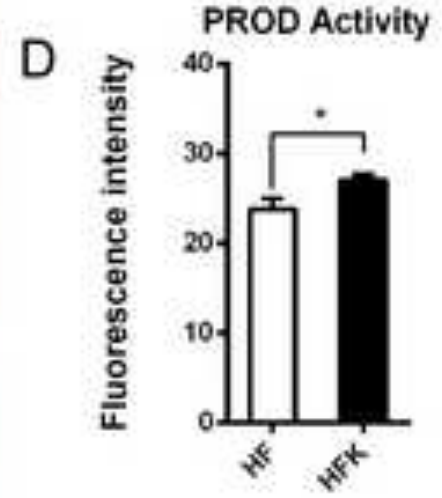
Alb Secretion

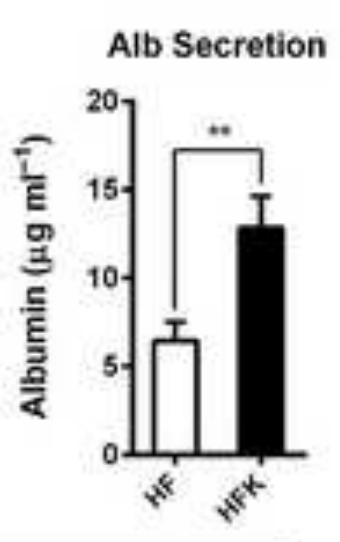

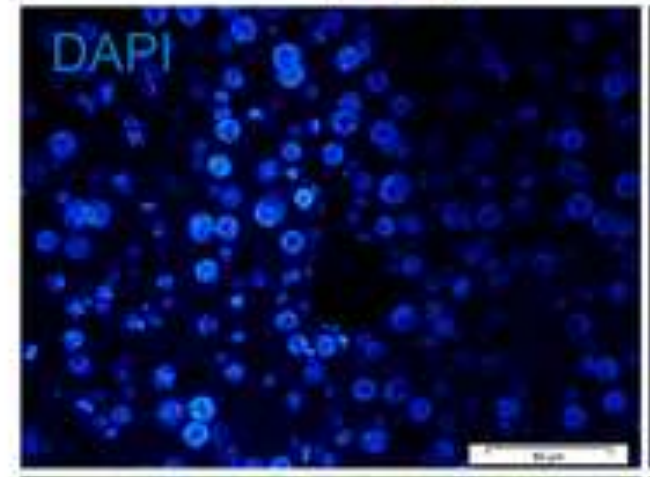

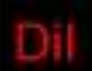

Alb

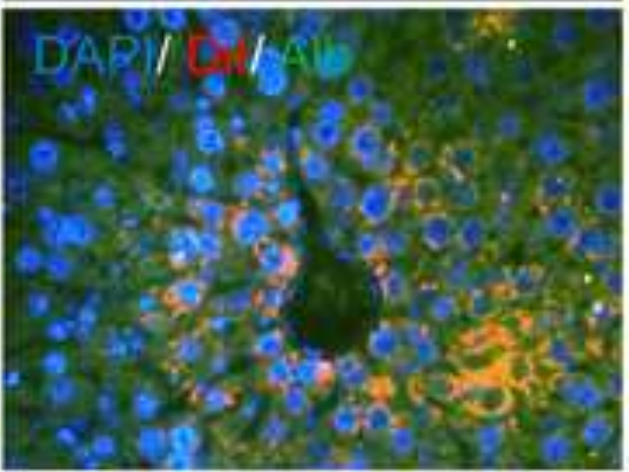

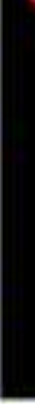

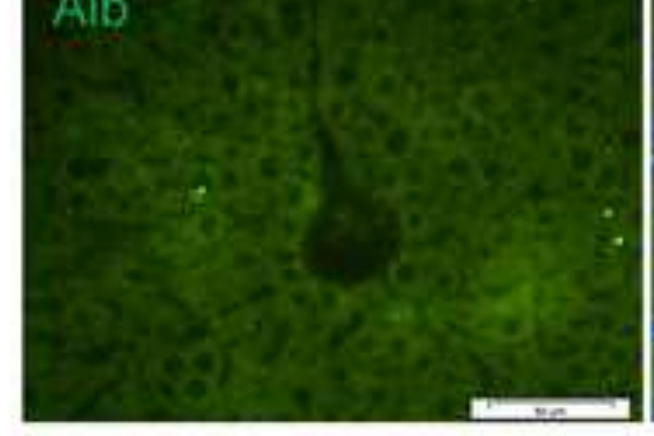




\section{Hepatic medium containing BMP4 and FGF4}

\section{Hnf4a and Foxa3 $\longrightarrow$}

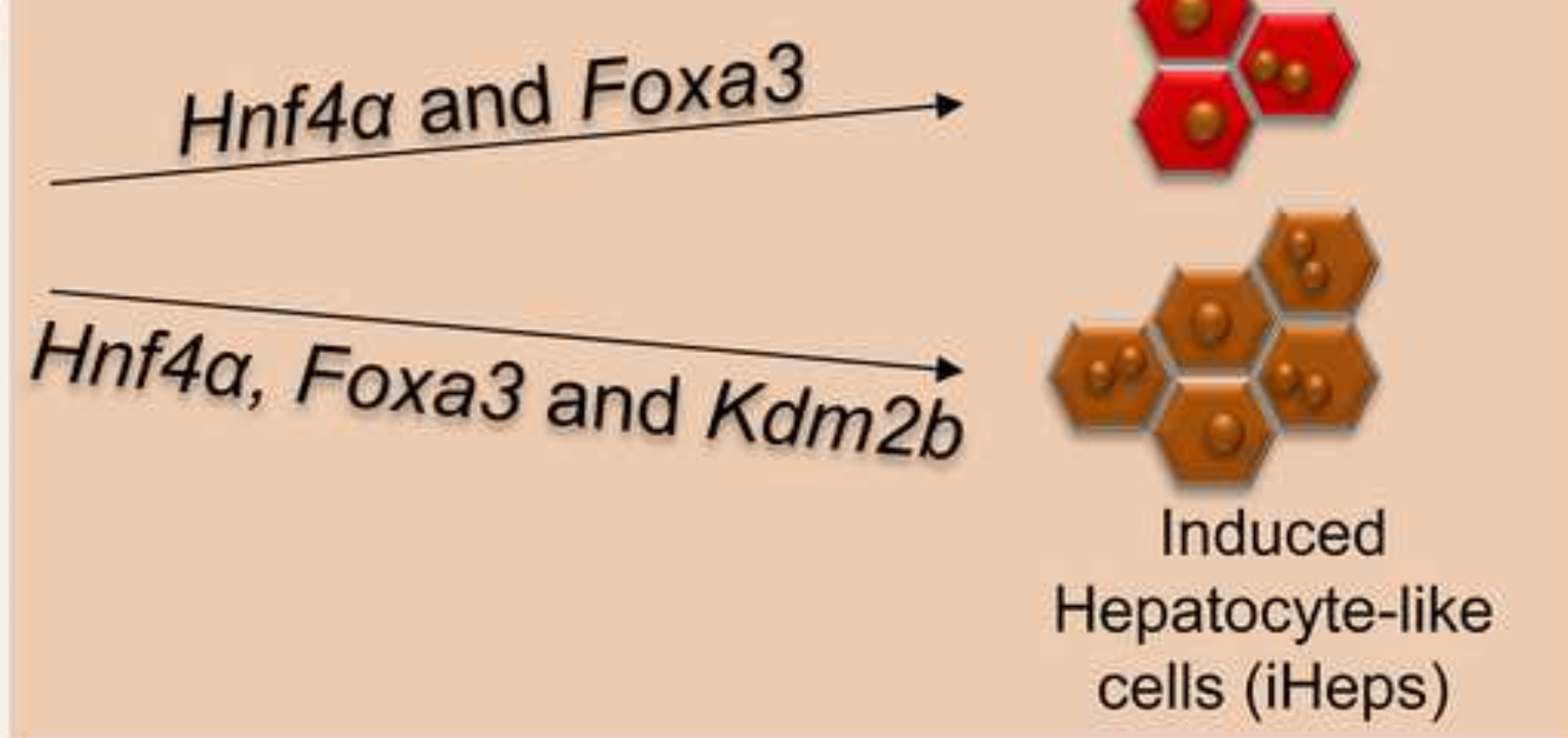

Fibroblasts

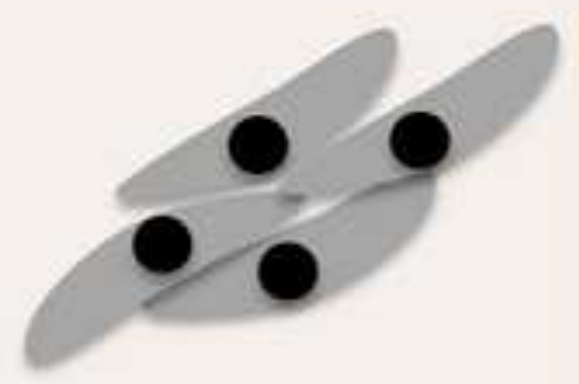

\section{Hnf4a, Foxa3 and $\mathrm{Kdm} 2 b$}

